\title{
An Association of Fragile X Associated Tremor/Ataxia Syndrome (FXTAS) with Three Common Neurological Problems: A Review
}

\author{
Shuja Yaqub, Robert SooHoo, Devyn Cottor, Hassaan Tohid* \\ Center for Mind and Brain, University of California, Davis, 95618, United States
}

*Corresponding author: Hassaan Tohid, Center for Mind and Brain, University of California, Davis, 95618, United States, E-mail: hassaantohid@hotmail.com

\begin{abstract}
Aim: To study the association of Fragile X associated tremor/ataxia syndrome (FXTAS) with Alzheimer's disease and Lewy Body Dementia.

Method: A systematic search was performed in various data bases and journals of Neurology. In total over 600 articles were reviewed and out of those, 39 were selected based on selection criteria.

Results: FXTAS is associated with Alzheimer's disease and Lewy Body dementia because so many symptomatic and pathophysiological similarities are found between FXTAS and these two neurodegenerative diseases.

Conclusion: The association of FXTAS with neurodegenerative illnesses like Alzheimer's disease and Lewy Body dementia is present because of many similarities between them. However, some dissimilarity does exist and some questions remain in addressing the pathophysiology of this association. Therefore we recommend more research in the near future to understand this association to develop and utilize wide-reaching therapies.
\end{abstract}

Received Date: Feb 26, 2016
Accepted Date: July 21, 2016
Published Date: July 25, 2016

Citation: Tohid, H., et al. An Association of Fragile X Associated Tremor/Ataxia Syndrome (FXTAS) with Three Common Neurological Problems: A Review. (2016) Int J Neurol Brain Disord 3(2): 67- 71.

DOI: $10.15436 / 2377-1348.16 .795$

Keywords: FXTAS; FXTAS Alzheimer's disease; FXTAS Alzheimer's Disease; FXTAS Alzheimer; FXTAS dementia; FXTAS memory; FXTAS symptoms; FXTAS Lewy Body dementia; Lewy body FXTAS.

\section{Introduction}

When the word "dementia" is discussed. Most people probably think of Alzheimer's disease (AD). However, there many other rare causes of dementia. One of these rare causes is Fragile X-associated tremor/ataxia syndrome (FXTAS). FXTAS is a rare neurodegenerative disease seen mostly in men aged 50 and above ${ }^{[1]}$. One in 3000 men is affected by the syndrome. FXTAS arises in male and female carriers of a premutation expansion (55 to 200 CGG repeats) of the fragile X mental retardation 1 (FMR1) gene. Common symptoms include memory problems, behavioral instability and cognitive decline especially in executive function, memory and visuospatial function. These neurodegenerative symptoms are synchronized with cortical-subcortical dementia ${ }^{[2]}$. Other symptoms seen in FXTAS patients include tremors and ataxia, which ultimately affect tasks like handwriting and lead to balance problems, limb weakness, muscle rigidity, speech problems, and lack of response to stimuli (e.g. pin prick) ${ }^{[3]}$. Furthermore, bladder and bowel control could also be affected in FXTAS. The degeneration involved in FXTAS is confined mostly to white matter ${ }^{[4]}$.

Since the establishment of neuropsychiatry as a recognized sub-specialty of medicine, interest in diseases like FXTAS has increased, not least due to the association of neuropsychological symptoms like apathy, delusions, hallucinations, sleep problems and cognitive impairments with neurodegenerative diseases. Neuropsychiatric symptoms such as these are not exclusive to FXTAS and occur in other common disorders such as Alzheimer's, Parkinson's and Huntington's. These symptoms also present in 50\% to $80 \%$ patients with frontotemporal and Lewy body dementias.

In this review, we aim to relate fxtas in particular to AD and LBD. Because of the common symptoms observed in these conditions, it is conceivable that there is an association between the three conditions. Thus, this review will broaden our understanding of the possible association of these conditions with each other.

Copyrights: (C) 2016 Tohid, H. This is an Open access article distributed under the terms of Creative Commons Attribution 4.0 International License. 


\section{Method}

Comprehensive review of published literature was conducted in PubMed, EMBASE, Wiley Online Library, Science Direct, Neuropsychologia, Elsevier, PLoS Biology, Current Biology, Neuroscience and Biobehavioral Reviews, PNAS, Neurophysiology, Nature Neuroscience, EDGE, Trends in Cognitive Sciences, Science, Behavioral and Brain Sciences, Cambridge Journals, Journal of Consciousness Studies, Cell, Philosophical Transitions B, The Royal Society B: Biological Sciences, Cerebral Cortex, SAGE Journals, Psychnet, Psych ARTICLES, Journal of Cognitive Neuroscience, Frontiers in Systems Neuroscience, Brandeis University, Oxford Journals, Social Cognitive and Affective Neuroscience, Physiology and Behavior, Frontiers in Human Neuroscience, BMC Medicine, International Review of Neuroscience, Developmental Neuroscience, Neuroscience letters, and Scientific American, JAMA Neurology, Journal of Alzheimer's Disease, Journal of Clinical Neuroscience, Journal of Intellectual \& Developmental Disability, Journal of Neurology, Movement Disorders, Journal of Neurology, Neurosurgery, and Psychiatry, Journal of Neuropathology \& Experimental Neurology, Current Alzheimer Research, Current Opinion in Neurology, European Journal of Neurology, Experimental Neurology, Annals of Neurology, The American Journal of Alzheimer's Disease \& Other Dementias, Alzheimer's Research \& Therapy, Alzheimer Disease and Associated Disorders, Brain, Nature Reviews Neurology and journal of Neuromuscular Disorders, Acta Neurologica Belgica, Acta Neurologica Scandinavica, Acta Neuropathologica, Acta Neuropsychiatrica and Journal of the Neurological Sciences.

No date restrictions were used. Articles included were those relevant to the history of FXTAS, Fragile X and FXTAS, FXTAS and Alzheimer's disease, and FXTAS and Lewy Body association. Keywords included but were not limited to: .FXTAS, FXTAS Alzheimer's disease, FXTAS Alzheimer's Disease, FXTAS Alzheimer, FXTAS dementia, FXTAS memory, FXTAS symptoms, FXTAS Lewy Body dementia, Lewy body FXTAS.

Reference sections were reviewed for additional articles. Titles and abstracts were assessed to determine if an article was a relevant source to our thesis. In total, over eight hundred article titles and abstracts were reviewed; 39 articles were chosen for inclusion in the final paper. Data was obtained from descriptions of research projects, experiments, program evaluations, systematic reviews, and case series.

\section{Discussion}

\section{Alzheimer's disease and FXTAS}

Patients with FXTAS dementia have been compared with patients with Alzheimer's disease (AD) ${ }^{[5]}$. Some similarities and dissimilarities have been found between the two medical conditions. For example, language performance is higher in FXTAS than in AD patients due to the involvement of both parietal lobes in $\mathrm{AD}$, which ultimately causes impairment in language. Language and speech are not affected for the majority of FXTAS patients, however a cerebellar type of dysarthria is found in some patients with advanced disease ${ }^{[6]}$. Also evidence suggests that FXTAS patients score poorly compared to normal controls on Block Design ${ }^{[7,8]}$.
Seritan et al 2008 confirmed that the measures of executive functions like verbal fluency, are expected to show reduced values in FXTAS, with more frontal involvement. They also found that FXTAS patients performed far better on a measure of simple attention. They also found that there was no significant difference between the working memory scores of FXTAS dementia patients and $\mathrm{AD}$ patients. This demonstrated that the working memory impairment in FXTAS dementia patients is not significantly different than the typical shortfalls seen in AD patients $^{[5]}$. While according to Grigsby et $\mathrm{a}^{[6]}$ attentional control impairment is found in some FXTAS patients when compared with AD patients. They also studied that FXTAS patient with cognitive impairment demonstrated moderate to severe shortfalls in working memory ${ }^{[6]}$.

Some gender differences have been found in FXTAS patients, according to two studies on female carriers of the FMR1 premutation. Decreased cerebellar volume and higher severity of FXTAS symptoms and increased length of the CGG repeat expansion were found in male premutation carriers only and not in females. However in women with FXTAS, the cognitive deficit is moderate in intensity and milder brain changes on MRI are observed as compared to their male counterparts ${ }^{[9,10]}$. This could be due to the protective effect of the second X chromosome or estrogen in females ${ }^{[1]}$.

Dementia with FXTAS has a cortical-subcortical pattern, because of the involvement of cortical (hippocampal, frontal) and subcortical (middle cerebellar peduncles, white matter) regions (95).

\section{Neurobiology of Alzheimer's disease and FXTAS}

Alzheimer's disease is another very prevalent neurodegenerative disease associated with cognitive dysfunction. Caused by protein plaques and neurofibrillary tangles that destroy the synaptic pathways and affect the neuronal connections. The main proteins involved in this disease are amyloid peptide protein (APP) and the tau protein. This process is caused by secretase enzymes ( $\beta$ and $\gamma$ ) which are located on cell membranes and form $\beta$-amyloids that are released into the plasma and cerebrospinal fluid where they aggregate and form plaques ${ }^{[12]}$. The formation of these plaques results in a breakdown of the synaptic microtubules and hyper phosphorylation of tau proteins. In FXTAS, cognitive degeneration is primarily caused by the mutation of the fragile X mental retardation gene (FMR1) which results in mediated RNA toxicity and defected mRNA. This can affect the brain's motor and cognitive functions. FMR1 in relation to Alzheimer's disease was studied by Abigail J. Renoux et al 2014[13] where FMRP (fragile X mental retardation protein FMRP, a protein constructed under the instruction of FMR1 gene) was found to have little expression in brains of AD patients. This suggests that there is little involvement of FMRP in AD neuropathology. However, similarities between AD and FXTAS have been discovered in that both neurodegenerative diseases involve APP and tau protein aggregates. Neuropathological evidence of both FXTAS eosinophilic inclusions and neurofibrillary tangles and neuritic plaques, consistent with $\mathrm{AD}$ are found ${ }^{[14]}$.

Due to the significant involvement of proteins in both neurodegenerative disorders Sokol et al 2011 ${ }^{[15]}$ carried out a study that examined the presence and link of APP FMRP between the diseases. This was conducted by using FMR1 knockout mice which exhibited high quantities of APP translation. 
This suggests that the FMR1 mutations can result in increased APP levels and may explain future cognitive deficits. Although the study focused on the Fragile X syndrome specifically, the analysis of FMR1 mice could be linked with FXTAS as well, as FXTAS is also associated with FMR1 and FMRP. The study suggested that amyloid plaques did not form due to the lack of FMR1 gene in the knock-out mice. However, in FXTAS as the disease is caused by elevation of FMR1-mRNA as stated by Kathryn Lovell et al $2008^{[16]}$, thus still enabling amyloidogenic processes to occur which could be a partial cause of cognitive impairment that befalls FXTAS patients.

Samples of the entorhinal cortex layer II of the medial temporal lobe from human brain showed decreased levels of 5-hydroxymethylcytidine $(5-\mathrm{hmC})$ and DNA (Cytosine-5-)-Methyltransferase 1 (DNMT1) in neurons of AD patients $^{[17]}$. 5-methylcytidine $(5-\mathrm{mC})$ and 5-hydroxymethylcytidine (5-hmC) were also studied by Chouliaras et al 2013. They found a similar reduction in $5 \mathrm{mC}$ and $5-\mathrm{hmC}$ in the hippocampal region of $\mathrm{AD}$ brains. Furthermore, levels of 5-mC as well as the levels of 5-hmC showed a significant negative correlation with amyloid plaque load in the hippocampus ${ }^{[18]}$. On the other hand a study also revealed opposite results, with augmented levels of $5 \mathrm{mC}$ and $5 \mathrm{hmC}$ in $\mathrm{AD}$ brains. These high levels of $5 \mathrm{mC}$ were seen in frontal cortex of AD patients ${ }^{[19]}$.

Another study revealed augmented levels of Ten-eleven translocation methylcytosine dioxygenase 1 (TET1), 5mC, and $5 \mathrm{hmC}$, with reduction in the levels of 5-formylcytosine $(5 \mathrm{fC})$ and 5-carboxylcytosine $(5 \mathrm{caC})$, in the hippocampus of AD patients, with no changes in cerebellum ${ }^{[20]}$. Coppieterts et al (2014) also observed increased levels of $5 \mathrm{mC}$ and $5 \mathrm{hmC}$ in the temporal and frontal cortex of the brain of AD patients ${ }^{[21]}$. The APP and Presenilin 1 (PSEN1) genes have also shown Alzheimer disease related changes in $5 \mathrm{mc}$ levels in the previous studies ${ }^{[22,23]}$. Global levels of $5 \mathrm{hmC}$ in the rCGG mouse model of FXTAS have been found, which shows the over expression of human CGG repeats within the 5 UTR of the FMR1gene in Purkinje neuronal cells, which ultimately leads to cellular death and behavior defi$\operatorname{cits}^{[24]}$. Yao B et al (2014), showed a genome-wide reduction in $5 \mathrm{hmC}$ levels in the cerebellum of rCGG mice compared with age matched wild-type controls, mainly within gene bodies and GC dinucleotides, termed $\mathrm{CpG}$ islands (CGIs) ${ }^{[25]}$. According Hall et al (2014) FMR1 is not associated with Alzheimer's disease ${ }^{[26]}$. Instead some studies show that AD and FXTAS both have mitochondrial dysfunction and oxidative stress and these problems could be amplified when they occur together ${ }^{[27-30]}$.

\section{Symptoms}

The summarized report of FXTAS produced by Hagerman RJ et al $2015^{[1]}$ also included in depth analysis of the cognitive deficits and impairments associated with the disease. Various features of the FXTAS phenotype include mild cognitive impairment, behavioral instability, and cortical-subcortical dementia, both of which relate to the features of Alzheimer's disease. These common features sometimes affected the true diagnosis of the disorder. Features of cognitive impairment include loss of short term memory and reduced competence in memory and executive functions as well as various behavioral deficits such as attention and aggression.

Dementia within FXTAS patients can occur both atypically and gradually. It also occurs in succession with other disorders such as tremors and ataxia. An investigation by Philip

K. Mothersead et al $2005^{[14]}$ identified a 61 year old man with gradual neurodegeneration beginning with ataxia and tremors and progressing to cognitive deficits such as loss of short term memory as well as an inability to carry on a conversation. The patient also suffered severe injuries related to his gait ataxia such as a broken arm or leg. The man was examined using a number of tests such as the Wechsler Adult Intelligence test - III and Wechsler Memory Scale - III in which scores were significantly below normal levels. After detailed cognitive examination the patient was also listed as a research subject at the University of California-Davis within the MIND institute. The patient had significant ataxic tremor and cognitive impairments such as confusion of his left and right as well as behavioral deficits such as anxiety and obsessive compulsive behavior. This phenotype was significantly similar to the FXTAS phenotype in that the patient exhibited both tremors and ataxia as well as significant cognitive degeneration. The patient's brain MRI revealed signal intensities in the cerebellar peduncles and cerebral atrophy previously noted as an important trait in a study conducted by Chaussenot et al $2008^{[31]}$. The patient's CGG repeat was also within the premutation range at 93 and upon discovering that he was a grandparent of children with FXS the man was diagnosed with FXTAS. Overall, the subject presented a neuropsychological and physical decline from an intelligent individual in a rare case where the patient had both Alzheimer's disease and FXTAS thus resulting in rapid decline by dementia.

\section{Diagnosis}

A study conducted by Sachdev et al 2013 ${ }^{[32]}$ suggested that Alzheimer's is not exclusive to the gray matter of the brain but in fact affects the white matter of the brain as well. As a result, the experiment carried out by Filley CM et al 2015[33] examined white matter disease and cognitive impairment within carriers of the FMR1 premutation. White matter mainly consists of myelin, which is a layer of fat used to augment connectivity between nerve fibers. Thus, the degeneration of white matter in Alzheimer's can suggest that processing speed is slower. The investigation included 13 premutation carriers compared with 7 healthy controls that were examined using magnetic resonance spectroscope (MRS) and diffusion tensor imaging (DTI). Areas of interest included the middle cerebellar peduncles and corpus callosum, both areas of white matter and cognitive function. The study used performances of the Controlled Oral Word Association Test (COWAT), Symbol Digit Modalities Test and Behavioral Dyscontrol Scale and correlated the results with fractional anisotropy (FA) acquired from DTI. FA examines the connectivity (processing speed) of the brain. The results revealed that FXTAS patients had the lowest mean FA, suggesting that FXTAS results in cognitive deficits and reduced processing speed within the brain similar to AD, and also correspond to how white matter disease results in reduced connectivity of the brain. This can perhaps enable easier diagnosis of both FXTAS and Alzheimer's as DTI and FA would be able to reveal signs of white matter disease.

In relation to changes within the forebrain within patients of Alzheimer's an experiment conducted by Pedro T et al $2012^{[34]}$ examined the volumetric brain changes in the key areas of the cerebrum such as the thalamus, corpus callosum and medial temporal structures. The study revealed that the volumetric changes that lead to structural atrophy of the thalamic areas provide a distinct indicator for mild $\mathrm{AD}$ to be identified. As a result, 
when Adams JS et al $2007^{[10]}$ carried out their experiment concerning females with FXTAS and the volumetric brain changes associated with them they discovered that the females demonstrated a less brain parts involvement in comparison with males who were also affected. The patients also followed consistent patterns of brain atrophy and white matter disease which can be linked to features of Alzheimer's as previously represented by Filley CM et al $2015^{[33]}$.

Early diagnoses of Alzheimer's and FXTAS aren't exclusively determined by volumetric expansions and examinations of the white matter. Another way to distinguish cognitive deficits and features is through messenger-ribonucleic acid (mRNA), which are single stranded RNA molecules 22 nucleotides in length. They work by suppressing protein genesis and are involved in a wide range of processes such as cell cycle control, stem cell differentiation and neurogenesis. Despite the numerous functions mRNA is also expected to be a future potential biomarker for Alzheimer's disease ${ }^{[35]}$. However, future studies possibly in the next quarter of the 21 st century will provide more details whether it could be used as a potential biomarker or not. The study detailed the many functions of mRNA and specifically examined the impacts on Alzheimer's as well as other neurological disorders. Areas affected by AD pathology are cortex, hippocampus, and the cerebellum. Moreover, the investigation also regarded the fragile $\mathrm{X}$ mental retardation diseases as effective demonstration of mRNA dysfunction in relation to the disorder. The mRNA is required for regulating synaptic plasticity as well as maintaining dendrite development. However, an alteration or absence of mRNA results in the deactivation of the Dicer enzyme (essential for RNA synthesis) in the Drosophila fruit fly, hence leads to tau-induced degeneration which also causes in Alzheimer's disease via neurofibrillary tangles leading to subsequent cognitive impairments.

\section{Lewy body dementia and FXTAS}

Lewy body dementias are specifically caused by lewy bodies which are irregular accumulations of protein that form within the nerves. The buildup of these proteins results in neurodegeneration resulting in Parkinsonism and cognitive impairment $^{[36]}$.

FXTAS could be associated with Lewy body according to some studies ${ }^{[37,38]}$. In spite of the possible similarities of the Lewy Body Dementia phenotype, some evidence from the study by Loesch et al $2015^{[39]}$ suggests that unlike similarities found within pathogeneses of some diseases for example, tau proteins in Alzheimer's; the pathogenesis for some FXTAS cases is different from cytoplasmic inclusions which are mainly associated with Lewy Body Dementia. The study also focuses on particular cases of FXTAS where a patient has contracted atypical forms of Parkinsonism as well as dementia but not the extreme forms such as mild cognitive impairment and ataxia. This irregular occurrence can be described as the "FXTAS spectrum" where some features of the syndrome overlap with other disorders such as Parkinson's with Lewy Body Dementia.

In the report summarizing the emerging topics in FXTAS Deborah A. Hall et al 2014 ${ }^{[7]}$ many cognitive disorders were identified specifically within older people. These disorders were able to co-exist within the patient, as post-mortem examinations of these subjects revealed that they not only had multiple sclerosis \& AD but also Lewy Body Dementia. This can suggest how disorders can synchronize within patients that have FXTAS. It is possible, that these issues were only discovered as a result of the autopsy of the patients. The features of parkinsonism and Alzheimer's disease as described by Hagerman et al 2015, Filley CM et al 2015, Andreea L. Seritin et al $2010^{[1,2,5]}$ are potentially difficult to distinguish as they bare close similarity to other diseases.

\section{Conclusion}

Fragile X-associated tremor/ataxia syndrome (FXTAS) is a rare neurodegenerative disease associated with memory problems, behavioral instability and cognitive decline, especially in executive and visuospatial abilities. Research exists which shows that patients with FXTAS may exhibit symptoms commonly seen in Alzheimer's disease and Lewy Body dementia. This mini review highlights the fact that these three neurological disorders (AD, LBD and FXTAS) can overlap in symptoms and somewhat in neurobiology: especially in that all of these disorders present with dementia. We believe articles like these will help future clinicians and scientists explore the unidentified aspects of any possible association. Further research is needed as there is more about dementia in these disorders that is unknown, and as new research is conducted it will broaden our understanding of dementia not only in FXTAS, LBD \& AD, but about all possible causes of dementia.

\section{Acknowledgements}

The authors are grateful for the help, support and suggestions by Dr. Jin Chen Yang (UC Davis). 


\section{References}

1. Hagerman, P.J, Hagerman, R.J. Fragile X-associated tremor/ataxia syndrome.(2015) Ann N Y Acad Sci 1338: 58-70.

2. O'Keefe, J.A, Robertson-Dick E., Dunn, E.J. Characterization and Early Detection of Balance Deficits in Fragile X Premutation Carriers With and Without Fragile X-Associated Tremor/Ataxia Syndrome (FXTAS).(2015) Cerebellum 14(6): 650-662.

3. Hall, D.A., Howard, K., Hagerman, R., et al. Parkinsonism in FMR1 premutation carriers maybe indistinguishable from Parkinson disease. (2009) Parkinsonism Relat Disord 15(2): 156-159.

4. Filley, C.M., Brown, M.S., Onderko, K., et al. White matter disease and cognitive impairment in FMR1 premutation carriers. (2015) Neurology 84(21): 2146-2152.

5. Seritan, A.L., Nguyen, D.V., Farias, S.T., et al. Dementia in fragile X-associated tremor/ataxia syndrome (FXTAS): comparison with Alzheimer's disease. (2008) Am J Med Genet B Neuropsychiatr Genet 147B(7): 1138-1144.

6. Grigsby, J., Leehey, M.A,, Jacquemont, S., et al. Cognitive impairment in a 65-year-old male with the fragile X-associated tremor-ataxia syndrome (FXTAS). (2006) Cogn Behav Neurol 19(3): 165-171.

7. Grigsby, J., Brega, A.G., Leehey, M.A., et al. Impairment of executive cognitive functioning in males with fragile $\mathrm{X}$-associated tremor/ ataxia syndrome. (2007) MovDisord 22(5): 645-650.

8. Grigsby, J., Brega, A.G., Engle, K., Leehey, M.A., et al. Cognitive profile of fragile $\mathrm{X}$ premutation carriers with and without fragile $\mathrm{X}$-associated tremor/ataxia syndrome.(2008) Neuropsychology 22(1): 4860 .

9. Hagerman, R.J, Leavitt, B.R., Farzin, F., et al. Fragile-X-associated tremor/ataxia syndrome (FXTAS) in females with the FMR1 premutation. (2004) Am J Hum Genet 74(5): 1051-1056.

10. Adams, J.S., Adams, P.F., Nguyen, D., et al. Volumetric brain changes in females with fragile $\mathrm{X}$-associated tremor/ataxia syndrome (FXTAS). (2007) Neurology 69(9): 851-859.

11. Berry-Kravis, E., Potanos, K., Weinberg, D., et al. Fragile X-associated tremor/ataxia syndrome in sisters related to X-inactivation. (2005) Ann Neurol 57(1): 144-147.

12. Seeman, P., Seeman, N. Alzheimer's disease: $\beta$-amyloid plaque formation in human brain.(2011) Synapse 65(12):1289-1297.

13. Renoux, A.J., Carducci, N.M., Ahmady, A.A., et al. Fragile X mental retardation protein expression in Alzheimer's disease. (2014) Front Genet 21: 5-360.

14. Mothersead, P.K., Conrad, K., Hagerman, R.J., et al. An atypical progressive dementia in a male carrier of the fragile $\mathrm{X}$ premutation: An example of fragile X-associated tremor/ataxia syndrome. (2005) ApplNeuropsychol 12(3): 169-178.

15. Sokol, D.K., Maloney, B., Long, J.M., et al. Autism, Alzheimer disease, and fragile X: APP, FMRP, and mGluR5 are molecular links. (2011) Neurology 76(15): 1344-1352.

16. http://learn.chm.msu.edu/NeuroEd/neurobiology_disease/content/ otheresources/fragileX.pdf

17. Mastroeni, D., Grover, A., Delvaux, E., et al. Epigenetic changes in Alzheimer's disease: decrements in DNA methylation. (2010) Neurobiol Aging 31(12): 2025-2037.

18. Chouliaras, L., Mastroeni, D., Delvaux, E., et al. (2013). Consistent decrease in global DNA methylation and hydroxymethylation in the hippocampus of Alzheimer's disease patients. (2013) Neurobiol Aging 34(9): 2091-2099.

19. Rao, J.S., Keleshian, V.L., Klein, S., et al. Epigenetic modifications in frontal cortex from Alzheimer's disease and bipolar disorder patients. (2012) Transl Psychiatry 2: e132.
20. Bradley-Whitman, M.A., Lovell, M.A. Epigenetic changes in the progression of Alzheimer's disease. (2013) Mech Ageing Dev 134(10): 486-495.

21. Coppieters, N., Dieriks, B.V., Lill, C., et al. (2014). Global changes in DNA methylation and hydroxymethylation in Alzheimer's disease human brain.(2014) Neurobiol Aging 35(6): 1334-1344.

22. Rogaev, E.I., Lukiw, W.J., Lavrushina, O., et al. The upstream promoter of the beta-amyloid precursor protein gene (APP) shows differential patterns of methylation in human brain. (1994) Genomics 22(2): 340-347.

23. Tohgi, H., Utsugisawa, K., Nagane, Y., et al. Reduction with age in methylcytosine in the promoter region -224 approximately -101 of the amyloid precursor protein gene in autopsy human cortex.(1999) Brain Res Mol Brain Res 70(2): 288-292.

24. Hashem, V., Galloway, J.N., Mori, M., et al. Ectopic expression of CGG containing mRNA is neurotoxic in mammals. (2009) Hum Mol Genet 18(13): 2443-2451

25. Yao, B., Lin, L., Street, R.C., et al. Genome-wide alteration of 5-hydroxymethylcytosine in a mouse model of fragile X-associated tremor/ ataxia syndrome. (2014) Hum Mol Genet 23(4): 1095-1107.

26. Hall, D.A., Bennett, D.A., Filley, C.M., et al. Fragile X gene expansions are not associated with dementia. (2014) Neurobiol Aging 35(11): 2637-2638

27. Ross-Inta, C., Omanska-Klusek, A., Wong, S., et al. Evidence of mitochondrial dysfunction in fragile $\mathrm{X}$-associated tremor/ataxia syndrome. (2010) Biochemical J. 429(3): 545-552.

28. Napoli, E., Ross-Inta, C., Wong, S., et al. Altered zinc transport disrupts mitochondrial protein processing/import in fragile $\mathrm{X}$-associated tremor/ataxia syndrome. (2011) Hum Mol Genet 20(15): 3079-3092.

29. Ye, X., Tai, W., Zhang, D. The early events of Alzheimer's disease pathology: from mitochondrial dysfunction to BDNF axonal transport deficits. (2011) Neurobiol Aging 33(6): 1122.el-10.

30. Karbowski, M., Neutzner, A. Neurodegeneration as a consequence of failed mitochondrial maintenance.(2012) ActaNeuropathol 123(2): 157-171.

31. Chaussenot, A., Borg, M., Bayreuther, C., Lebrun, C. [Late cerebellar ataxia associated with fragile X premutation]. (2008) Rev Neurol (Paris) 64(11): 957-963.

32. Sachdev, P.S., Zhuang, L., Braidy, N., et al. Is Alzheimer's a disease of the white matter? (2013) CurrOpin Psychiatry 26(3): 244-251.

33. Filley, C.M., Brown, M.S., Onderko, K., et al. White matter disease and cognitive impairment in FMR1 premutation carriers.(2015) Neurology 84(21):2146-2152.

34. Pedro, T., Weiler, M., Yasuda, C.L., et al. Volumetric brain changes in thalamus, corpus callosum and medial temporal structures: mild Alzheimer's disease compared with amnestic mild cognitive impairment. (2012) Dement GeriatrCognDisord 34(3-4): 149-55.

35. Maes, O.C., Chertkow, H.M., Wang, E., et al. MicroRNA: Implications for Alzheimer Disease and other Human CNS Disorders. (2009) Curr Genomics 10(3): 154-168.

36. McKeith, I. Dementia with Lewy bodies. (2004) Dialogues ClinNeurosci 6(3): 333-341

37. McKeith, I.G. Consensus guidelines for the clinical and pathologic diagnosis of dementia with Lewy bodies (DLB): report of the Consortium on DLB International Workshop. (2006) J Alzheimers Dis 9(3 suppl): 417-423.

38. Greco, C.M., Hagerman, R.J., Tassone, F., et al. Neuronal intranuclear inclusions in a new cerebellar tremor/ataxia syndrome among fragile X carriers. (2002) Brain 125(Pt 8): 1760-1771.

39. Loesch, D.Z., Bui, M.Q., Hammersley, E., et al. Psychological status in female carriers of premutation FMR1 allele showing a complex relationship with the size of CGG expansion.(2015) Clin Genet 87(2): 173-178.

\section{Ommega Online Publishers}

E-mail: neurology@ommegaonline.com

Website: www.ommegaonline.org
Journal Title: International Journal Neurology and Brain Disorders Journal Short Name: Int J Neurol Brain Disord 